\title{
Reduction of Fluid Forces and Vortex Shedding Frequency of a Circular Cylinder using Rigid Splitter Plates
}

\author{
Rezvan Abdi ${ }^{* a}$, Niki Rezazadeh ${ }^{\mathrm{a}}$, Meisam Abdi $^{\mathrm{b}}$ \\ *Rezvan_Abdi@yahoo.com \\ ${ }^{a}$ Faculty of Engineering, Hakim Sabzevari University, Sabzevar, Iran \\ ${ }^{b}$ Faculty of Engineering, University of Nottingham, Nottingham, UK
}

\begin{abstract}
This study investigates the fluid forces acting on a circular cylinder in a laminar flow regime while using a passive control strategy. Three cases including the cylinder with one, two or three rigid splitter plates attached at its rear surface were considered and the location of horizontal plates (attachment angle) was varied between $0^{\circ}$ and $90^{\circ}$. A comprehensive parametric study was performed to identify the optimum arrangement of the plates using the commercial finite element software, Comsol Multiphysics. The results show that the location and the number of the plates have crucial effects on the wake control. Increasing the number of splitter plates from one to two symmetric parallel plates led to a reduction in drag force, vortex shedding frequency and fluctuation of lift force. A maximum drag reduction of $23 \%$ for dual-splitters and $15 \%$ for single-splitter was achieved, at an angle of $45^{\circ}$ at Reynolds number 100. However, increasing the number of attached plates to three didn't have a significant effect on flow quantities when plates of the same length were utilized. The suitability of the third plate (the middle plate) was further studied by investigating the effect of length of the plate on flow quantities.
\end{abstract}

Keywords: rigid splitter plate, vortex shedding, drag reduction, laminar flow, circular cylinder, Strouhal number.

\section{Introduction}

As flow passes over a bluff body, two recirculating zones, known as vortices, are formed downstream of the bluff body. When Reynolds number (Re), of the flow exceeds a threshold number, the vortices start to peel off periodically from the cylinder. When the vortex shedding occurs behind a cylinder, drag on the cylinder increases. Moreover, the periodic forces that were applied on the cylinder in the cross flow direction cause undesirable vibrations in the cylinder (Kwon and Choi, 1996). Many practical applications involve vortex-induced vibrations, including heat exchanger tube bundles, marine structures, bridges, power transmission lines etc. Therefore, controlling the vortex shedding is very important in engineering applications to prevent the possible structural damages (Sudhakar and Vengadesan, 2012).

Previous works on the wake control of vortex shedding from a circular cylinder can be classified into two categories including active control and passive control. In the active control strategy, an input energy is required to be applied in the form of suction or blowing into the base region of the cylinder in order to control/reduce vortex shedding. However, in passive control strategy, an input energy is not required; instead, modifications of either the surface or the near-wake flow of the cylinder using some devices enables the reduction of vortex shedding (Yucel et al, 2010). Among passive control approaches, the use of splitter 
plates has been one of the most successful methods which can lead to reduction and suppression of vortex street behind the cylinder (Kwon and Choi, 1996).

The splitter plates can be in the form of attached plates or detached plates. Early studies regarding the influence of splitter plates on cylinder wake was initiated by Roshko (1954). Roshko investigated the effect of splitter plate ratio $(L / D$, where $L$ is the plate length and $D$ is the cylinder diameter) on cylinder wake for a cylinder with an attached splitter plate. $\mathrm{He}$ reported that when the splitter plate was sufficiently long, e.g. $L / D \geq 5$, the vortex shedding was completely eliminated; hence, drag coefficient $\left(C_{D}\right)$ significantly reduced. The effect of splitter plate length was investigated by Gerrard (1966) at $\mathrm{Re}=2 \times 10^{4}$. He reported that increasing the length of the plate resulted in decreasing St within $0 \leq L \leq D$. However, St gradually increased by further increasing the plate length. Therefore, the minimum St was observed when the splitter plate ratio was approximately equal to one. He showed that the frequency of vortex shedding is inversely proportional to the vortex formation length $\left(L_{F}\right)$ immediately downstream of the bluff body. Similar experiments were conducted by Unal and Rockwell (1988) in the Re range of $140<\mathrm{Re}<1600$. They divided the wake region into prevortex formation region and post-vortex formation region and observed a change in the pressure on the control plate when the plate was shifted from one region to another. Apelt et al (1973) and Apelt and West (1975) performed an experiment to study the effect of length of splitter plate for $10^{4} \leq \operatorname{Re} \leq 5 \times 10^{4}$. It was reported that the drag decreased as the length of the plate was increased up to $L / D=1$. Then, it had an increasing trend within $1 \leq L / D \leq$ 2 , and after that, it decreased again. Apelt and West (1975) showed that adding the splitter plate led to a reduction in drag force and an enhancement in the base pressure. Moreover, similar trend was observed for the variation of $C_{D}$ in comparison with the St variation at a higher $\operatorname{Re}\left(=5 \times 10^{4}\right)$ in their experiments.

In addition to the above studies that considered attached splitters, there are studies that investigated the effect of detached splitter plates on cylinder wake. Roshko (1955) investigated the effects of adding detached splitter plate with splitter plate ratio of $L / D=1$. He observed an increase in base pressure and a decrease in Strouhal number (St) (a dimensionless number describing oscillating flow mechanisms) when splitter plate was fixed downstream at a distance less than $4 D$. Hwang et al (2003) investigated the effects of a detached splitter plate on a circular cylinder and observed a considerable reduction of flowinduced forces on the cylinder. Akilli et al (2005) extended the previous experimental studies to investigate the effects of detached splitter plate on the suppression of vortex shedding by varying the position of the plate. Ozono (2003) studied the effects of length and position of a detached splitter plate behind a cylinder at an asymmetric configuration. He observed that by increasing the gap between the cylinder base point and the leading edge of the downstream splitter plate, the values of St and $C_{D}$ initially started decreasing. Serson et al (2015) studied detached splitter plates and observed a strong dependence on Re, with the splitter plates being more beneficial at the higher values of Reynolds numbers.

More recently, Shukla et al (2009) investigated the influence of attaching a hinged-rigid splitter plate to a cylinder on cylinder wake. Shukla stated that the communication between the shear layers downstream of the cylinder was not disrupted completely with using the hinged splitter plate. Furthermore, the splitter plate oscillated due to the pressure difference across the plate and the amplitude of oscillations reached to a maximum value of $0.45 \mathrm{D}$ at the free end. Similar investigations regarding the use of hinged rigid splitter plate attached to a cylinder were performed by Assi et al (2009), and $\mathrm{Gu}$ et al (2012). There are also a few studies investigating the effect of a flexible splitter plate on flow quantities of the cylinder (Shukla et al, 2013; Sudhakar and Vengadesan, 2012; Tian et al, 2011; Nayer and Breuer, 2014). A number of recent studies have also investigated the use of dual-splitter plates in the 
wake control (Bao and Tao, 2013; Serson et al 2015; Qiu et al, 2014; Barman and Bhattacharyya 2015). Bao and Tao (2013) used short splitter plates of size $L / D=0.3$ for the wake control of vortex shedding. They also investigated the effect of length of the plates at a fixed position around the cylinder and observed that the length of the plates had crucial effects on drag reduction and wake suppression.

As discussed above, most of the previous works regarding the control and stabilisation of wake flow behind a cylinder only considered cylinders with one rigid splitter plate in a fixed angular position. The novelty of this paper is that it investigates the effects of having multiple rigid plates, attached to a cylinder on cylinder wake by comparing single-splitter, dualsplitters and tri-splitters cases. Moreover, the influence of the location of horizontal plates (attachment angle) on the cylinder wake is studied in the paper. This is followed by a more detailed study on the effect of the length of the middle plate in tri-splitters case on flow quantities. The same splitter plate ratio of $L / D=1$ which is greater than the length used in Bao and Tao (2013) study was initially considered for all the cases. The plates were located downstream of the cylinder and the variation of Strouhal number, drag coefficient and rootmean-square (r.m.s) value of the fluctuating lift coefficient was investigated. The effect of the length of the middle plate in tri-splitters case was also investigated to support the initial findings obtained for this arrangement at $L / D=1$. The commercial software, Comsol Multiphysics V.4.3a (COMSOL Inc., Stockholm, Sweden), was utilized to solve the problem using the finite element method.

The paper is organized as follows. The problem statement is given in section 2. The numerical methodology used to solve the equations and validation of the numerical simulation, are presented in section 3 . The detailed results and the discussion are presented in section 4 and final conclusions are given in section 5.

\section{Problem Statement}

In the present work, the problem of laminar flow past a circular cylinder is considered for three different cases: the cylinder with one, two and three rigid splitter plates attached to the rear surface of the cylinder as shown in figure 1. In all these cases, the thickness of the plates (h) was equal to $0.03 D$. The location of the horizontal plates behind the cylinder which was defined by attachment angle, $\theta_{p}$, (i.e., the angle between the wake centreline and a straight line crossing the centre of the cylinder and the attachment) was varied between $\theta_{p}=0^{\circ}$ and $\theta_{p}=90^{\circ}$, leading to an asymmetric configuration for single-splitter case. However, in the cases of dual-splitters and tri-splitters, symmetric configurations were assumed, as can be seen in figure 1.

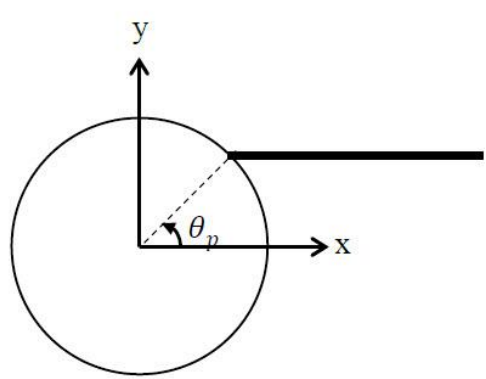

(a)

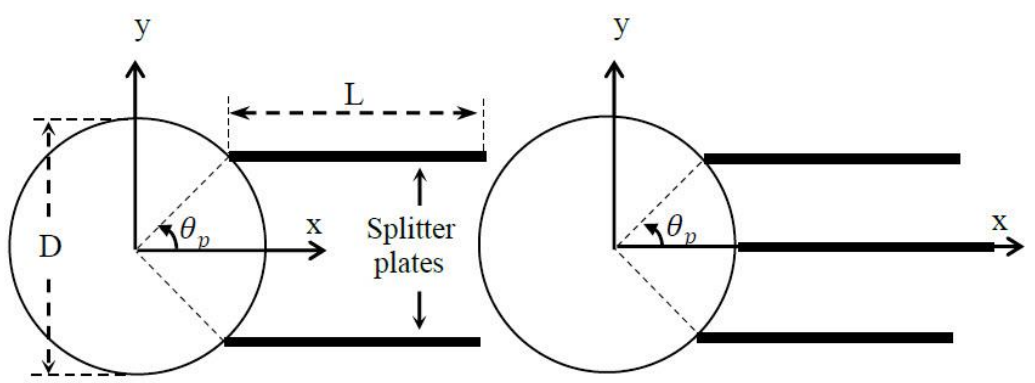

(c)

Figure 1: Schematic diagram of a cylinder with splitter plates.

(a) Single-splitter (b) dual-splitters (c) tri-splitters 


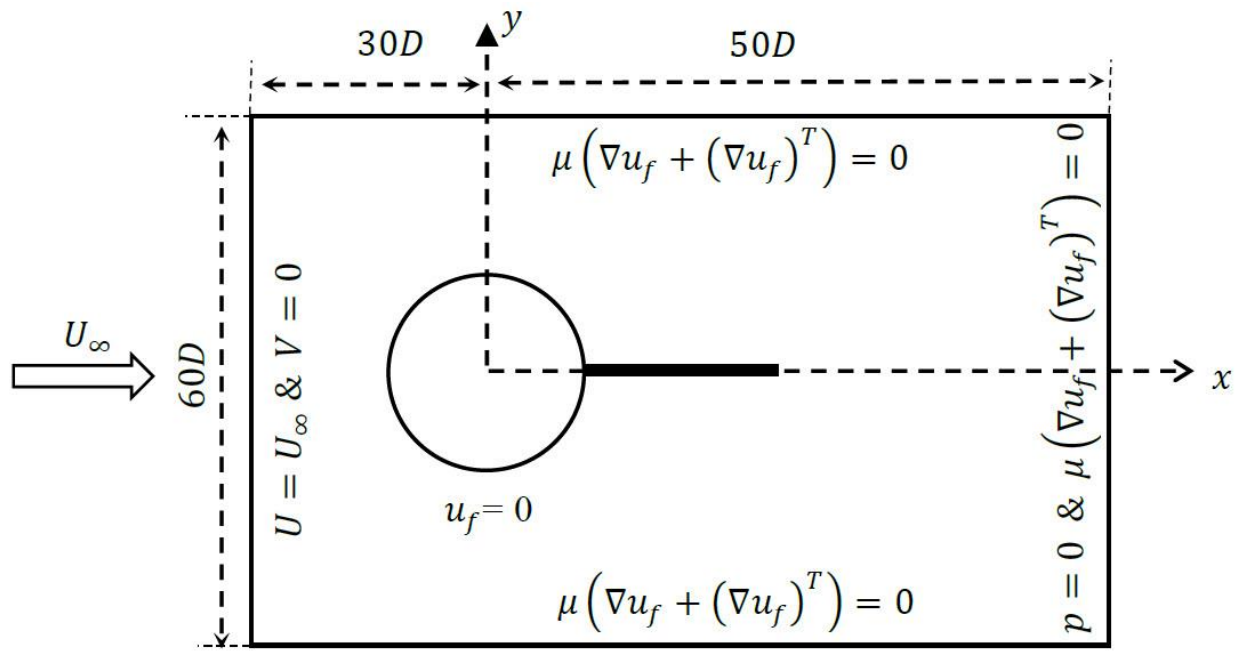

Figure 2: The computational domain and boundary conditions

The flow geometry and coordinate system as well as boundary conditions are shown in figure 2. The computational domain was defined within $-30 D \leq x \leq 50 D$ and $-30 D \leq y \leq 30 D$, where the centre of the cylinder was located at the origin $(x=0, y=0)$. The effect of the outlet boundary and the blockage effect were negligible in this problem as the size of the domain was large enough in both flow and cross-flow directions, respectively. The inlet velocity $U$ was uniform, aligned with the $x$-direction and was chosen such that $R e=$ $\rho U_{\infty} \mathrm{D} / \mu=100$. Pressure outlet boundary condition was applied on the far downstream outlet. Assuming the environment had the atmosphere conditions, a pressure value of zero was used on the outlet boundary. The open boundary conditions with no viscous stress were imposed to the lateral surfaces. A no-slip boundary condition specified as $u_{f}=0$ was applied on the cylinder wall and on the splitter plate, where $u_{f}$ is the fluid velocity. Glycerin with dynamic viscosity of $1.42 \frac{\mathrm{kg}}{\mathrm{m} \cdot \mathrm{s}}$ and density of $1260 \frac{\mathrm{kg}}{\mathrm{m}^{3}}$ was used in the fluid domain.

The Reynolds number is defined as $\operatorname{Re}=\rho U_{\infty} \mathrm{D} / \mu$, where $\rho$ and $\mu$ are the density and dynamic viscosity of the fluid respectively, $U_{\infty}$ is the free-stream velocity and $D$ is the diameter of the cylinder. Non-dimensional parameters, such as the drag coefficient $\left(C_{D}\right)$, the lift coefficient $\left(C_{L}\right)$, the Strouhal number $(S t)$ and the pressure coefficient $\left(C_{P}\right)$ are defined by:

$S t=\frac{f_{S} D}{U_{\infty}}$

where $f_{s}$ is the vortex shedding frequency.

$C_{D}=\frac{F_{D}}{0.5 \rho U_{\infty}^{2} D}$

$C_{L}=\frac{F_{L}}{0.5 \rho U_{\infty}^{2} D}$

where $\rho$ is the fluid density and $F_{D}$ and $F_{L}$ are the fluid forces (i.e., the pressure and viscosity forces) applied on the cylinder, in the streamwise and transverse directions, respectively.

$C_{P}=\frac{P-P_{\infty}}{0.5 \rho U_{\infty}^{2}}$ 
where $p$ is the static pressure on the cylinder surface and $P_{\infty}$ is the free-stream static pressure.

\section{The Numerical Methodology and Validation}

\subsection{Governing equations and numerical formulation in Comsol Multiphysics}

The fluid flow is described by the mass continuity equation ( equation 4) and incompressible Navier-Stokes equation (equation 5) (neglecting the volume forces):

$\nabla \cdot u_{f}=0$

$\rho\left(\frac{\partial u_{f}}{\partial t}+u_{f} \cdot \nabla u_{f}\right)=\nabla \cdot\left[-p I+\mu\left(\nabla u_{f}+\left(\nabla u_{f}\right)^{T}\right)\right]$

where $\rho$ is the fluid density, $p$ is the pressure, $u_{f}$ is the velocity vector of the fluid and $I$ is the unit diagonal matrix.

\subsection{Finite element scheme}

Comsol Multiphysics solves problems defined with Partial Differential Equations (PDEs) using Finite Element Method (FEM). In Comsol, Implicit Differential-Algebraic (IDA) solver is used to solve the system of equations which was attained from discretisation of the timedependent PDE. IDA uses variable-order variable-step-size backward differentiation formulas (BDF) to discretise a PDE (Brown et al, 1994; Hindmarsh et al, 2005; Comsol, 2012).

The finite element equation of the discretized time-dependent PDE problem is given by:

$0=L(U, \dot{U}, \ddot{U}, t)-N_{F}(U, t) \Lambda$

$0=M(U, t)$

where $U$ is the solution vector, $L$ is the residual vector, $M$ is the constraint residual, $N_{F}$ is the constraint force Jacobian and $\Lambda$ is a Lagrange multiplier.

A Newton solver is fitted into the IDA to solve the nonlinear system of equations which is generated due to the use of implicit time-stepping schemes. The attained equations which are linearised to be used in the Newton's iteration are given by:

$E \ddot{V}+D \dot{V}+K V=L-N_{F} \Lambda$

$N V=M$

where $V$ is the nodal displacement vector, $K=\frac{-\partial L}{\partial U}$ is the stiffness matrix, $D=\frac{-\partial L}{\partial \dot{U}}$ is the damping matrix, $E=\frac{-\partial L}{\partial \ddot{U}}$ is the mass matrix and $N$ is the constraint Jacobian matrix. A PARDISO (Parallel Direct Sparse Solver) solver is used by Newton solver to solve the resulting linear systems. PARDISO acts on general sparse linear systems of the form $A x=b$ and employs $L U$ factorization on the matrix $A$ to find the solution $x$ (Schenk and Gartner, 
2004; Schenk and Gartner, 2006; Comsol, 2012).

\subsection{Validation of the finite element model}

The simulation results were compared with experimental and numerical results from the literature for two benchmark cases at $R e=100$ : flow past a plain cylinder (the cylinder without any splitter plates), and flow past a plain cylinder with one splitter plate attached at $\theta_{p}=0^{\circ}$. As shown in Table 1, the simulation results closely match other numerical (Hwang et al. 2003; Sudhakar and Vengadesan, 2012; Park et al. 1998; Kwon and Choi, 1996; Posdziech and Grundmann, 2007, Bao and Tao, 2013) and experimental (Williamson, 1989) results. The small differences between the present results and the numerical results from literature could be attributed to the different numerical approaches which have been implemented in the studies. Other possible reasons for the discrepancy of the numerical results in the different studies can include the blockage effect, the proximity of the cylinder to the inlet and the low cylinder distance from the outlet.

Table 1: Comparison of characteristic quantities for flow past a circular cylinder with and without splitter plates.

\begin{tabular}{|c|c|c|c|c|c|c|c|c|c|}
\hline Test cases & $\begin{array}{c}\text { Flow } \\
\text { quantities }\end{array}$ & $\begin{array}{l}\text { Hwang } \\
\text { et al. } \\
\text { (2003) }\end{array}$ & $\begin{array}{c}\text { Sudhakar \& } \\
\text { Vengadesan } \\
\text { (2012) }\end{array}$ & $\begin{array}{l}\text { Park et } \\
\text { al } \\
(1998)\end{array}$ & $\begin{array}{c}\text { Kwon } \\
\text { \& } \\
\text { Choi } \\
(1996)\end{array}$ & $\begin{array}{l}\text { Willia- } \\
\text { mson } \\
(1989)\end{array}$ & $\begin{array}{l}\text { Posdziech } \\
\& \\
\text { Grundmann } \\
(2007)\end{array}$ & $\begin{array}{c}\text { Bao } \\
\& \\
\text { Tao } \\
(2013)\end{array}$ & Present \\
\hline \multirow{2}{*}{$\begin{array}{l}\text { Plain cylinder, } \\
\quad \operatorname{Re}=100\end{array}$} & $\overline{\overline{C_{D}}}$ & 1.34 & 1.37 & 1.33 & - & - & 1.32 & 1.335 & 1.33 \\
\hline & St & 0.167 & 0.165 & 0.164 & - & 0.164 & - & 0.164 & 0.164 \\
\hline \multirow{2}{*}{$\begin{array}{c}\text { Single plate } \\
\operatorname{Re}=100, \mathrm{~L} / \mathrm{D}=1\end{array}$} & $\overline{\overline{C_{D}}}$ & 1.17 & 1.174 & - & 1.18 & - & - & - & 1.161 \\
\hline & St & 0.137 & 0.139 & - & 0.137 & - & - & - & 0.136 \\
\hline
\end{tabular}

\subsection{Grid independence test}

Figure 3 shows the computational domain meshed using triangular and quadrilateral elements. Due to the high value of the velocity gradient near the cylinder wall and the plate wall, quadrilateral elements were used close to these regions. For the rest of the computational domain, triangular elements were used. The grid independence test was conducted on the flow past the cylinder with one splitter plate at $\theta_{p}=0^{\circ}$. Four different mesh resolutions were used to examine the spatial convergence (Table 2).

Comparing the numerical results in table 2 , it can be seen that the mesh resolutions in case 1 and case 2 were appropriate to accurately evaluate the flow past over the cylinder. As the grid resolution was refined from case 2 to case 3, more accurate result was observed. However, further increase in mesh density (i.e., from case 3 to case 4 ) didn't cause significant change on the numerical measurements. Therefore the mesh setting in case 3 which is computationally less expensive than case 4 was chosen for all subsequent simulations. A minimum mesh quality of 0.7323 in Comsol was used in this case for the fluid domain. 


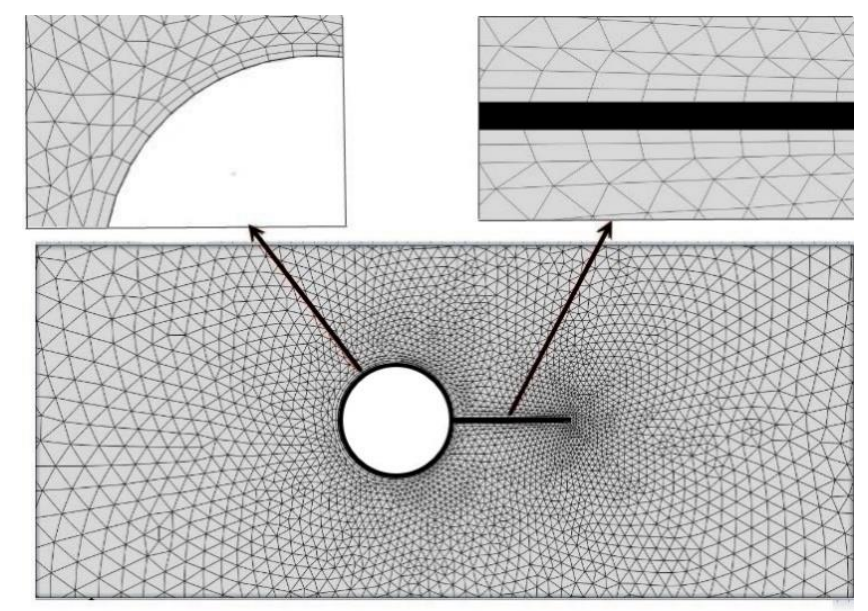

Figure 3: Mesh detail at the interface boundary between the plate wall and fluid domain

Table 2: Grid independence test for the flow past a circular cylinder with one rigid splitter plate attached at $\theta_{p}=0^{\circ}$.

\begin{tabular}{cccc}
\hline Case & Grid elements & $\overline{C_{D}}$ & St \\
\hline$(1)$ & 1069 & 1.164 & 1.149 \\
$(2)$ & 3450 & 1.162 & 1.144 \\
$(3)$ & 11976 & 1.161 & 1.144 \\
$(4)$ & 29495 & 1.161 & 1.144 \\
\hline
\end{tabular}

\section{Results and Discussion}

In this section, the effect of attaching one or more rigid splitter plates to the rear surface of the cylinder on Strouhal number $(\mathrm{St})$, mean drag coefficient $\left(\overline{C_{D}}\right)$ and fluctuating lift coefficient $\left(C_{L}\right)$ is investigated. The results are presented against the location of the plates behind the cylinder which is defined by attachment angle $(\theta p)$. In the case of cylinder with three attached splitters, the effect of the length of the plates is also investigated, focusing on the role of the middle plate in wake control.

\subsection{Vortex shedding frequency}

The Strouhal number (St) against attachment angle, $\theta p$, for flow past a cylinder with one, two or three rigid splitters is presented in figure 4. For comparison, St of the plain cylinder (the cylinder without any splitters) is also incorporated in the graph. The St was calculated from the period of the lift coefficient. It can be seen from figure 4 that the cylinder with one rigid splitter plate had lower value of St than the plain cylinder. This can be attributed to the fact that the reaction between the shear layers downstream of the cylinder is delayed by the use of the splitter plate. It can also be seen that in the case of cylinder with single-splitter plate, by increasing $\theta_{p}$ up to $15^{\circ}$, the $S t$ had a fixed value. However, by further increasing the attachment angle $\left(15^{\circ} \leq \theta_{p} \leq 90^{\circ}\right)$, St increased linearly. Therefore, a maximum shedding 
frequency reduction of $17 \%$ compared to the plain cylinder was achieved between $\theta_{p}=0^{\circ}$ and $\theta_{p}=15^{\circ}$ using the single-splitter.

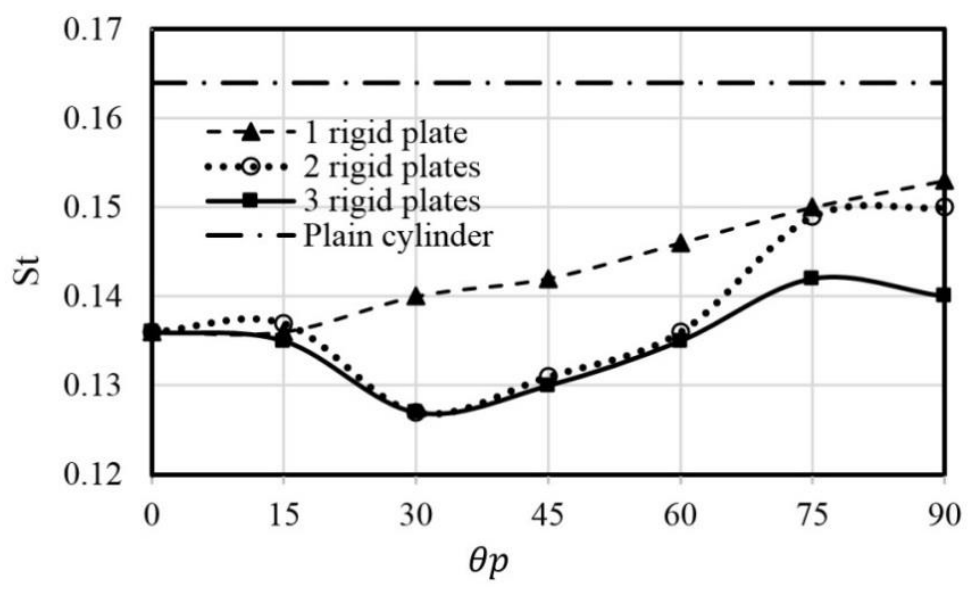

Figure 4: The variation of St number against the attachment angle in the three different splitter plate cases.

Figure 5 illustrates the instantaneous streamlines for the flow past a cylinder with singlesplitter (5a) and dual-splitters (5b). The increase in St occurred in single-splitter case within $15^{\circ} \leq \theta_{p} \leq 90^{\circ}$ can be explained by looking at figure 5(a). By increasing $\theta_{p}$, the upper vortex of the cylinder became narrower in the region close to the cylinder and grew in the region near the free end. On the other side, the lower vortex became larger and grew behind the cylinder under the plate (figure 5a). This asymmetry results in separation of the upper vortex (the vortex behind the tip of the plate). Following that, the lower vortex can grow and shed, and the process repeats itself. It can also be seen from figure 5 (a) that by increasing $\theta_{p}$ the asymmetry of vortex pairs with respect to the vortex positions, increased; thus, increasing St.

As can be seen from figure 4 , in the case of dual-splitters, St number remained constant between $\theta_{p}=0^{\circ}$ and $\theta_{p}=15^{\circ}$, and the change in St number became noticeable for $\theta_{p} \geq$ $15^{\circ}$. By increasing $\theta_{p}$ in the range of $15^{\circ} \leq \theta_{p} \leq 30^{\circ}$, a reduction in St number occurred. This is because of the fact that increasing the distance between the two plates leads to a delay in the contact between the shear layers. Following that, the vortex formation length increases, resulting in decreasing the St number (figure $5 \mathrm{~b}$ ). Then, by further increasing the attachment angle $\left(30^{\circ} \leq \theta_{p} \leq 90^{\circ}\right)$, the St number increased. This can be attributed to the reduction of vortex formation length as can be noticed from figure $5(\mathrm{~b})$. Similar trend can be seen for the tri-splitters up to $\theta_{p} \approx 75^{\circ}$ (figure 4 ). By further increasing $\theta_{p}$, the St number decreased due to the addition of the third plate (the middle plate). The middle plate prevented the growth of vortices in the region between the two plates; therefore, it led to a delay in contact between the vortices (figure 6). Generally, the St number of the cylinder with one or more splitter plates in all attachment angles was always lower than that of the plain cylinder. Moreover, the St numbers associated with the cylinder with dual-splitters and tri-splitters are lower than that of the single-splitter, especially within $20^{\circ}<\theta_{p}<70^{\circ}$. In the three different cases studied in this section, the lowest St was observed in cylinder with two/three splitter plates at $\theta_{p}=30^{\circ}$ in which the maximum shedding frequency reduction of $22 \%$ in comparison with plain cylinder was achieved. Therefore, comparing with the single-splitter case, adding the second splitter plate had a significant effect in reducing the shedding frequency and improving the wake stabilization. 


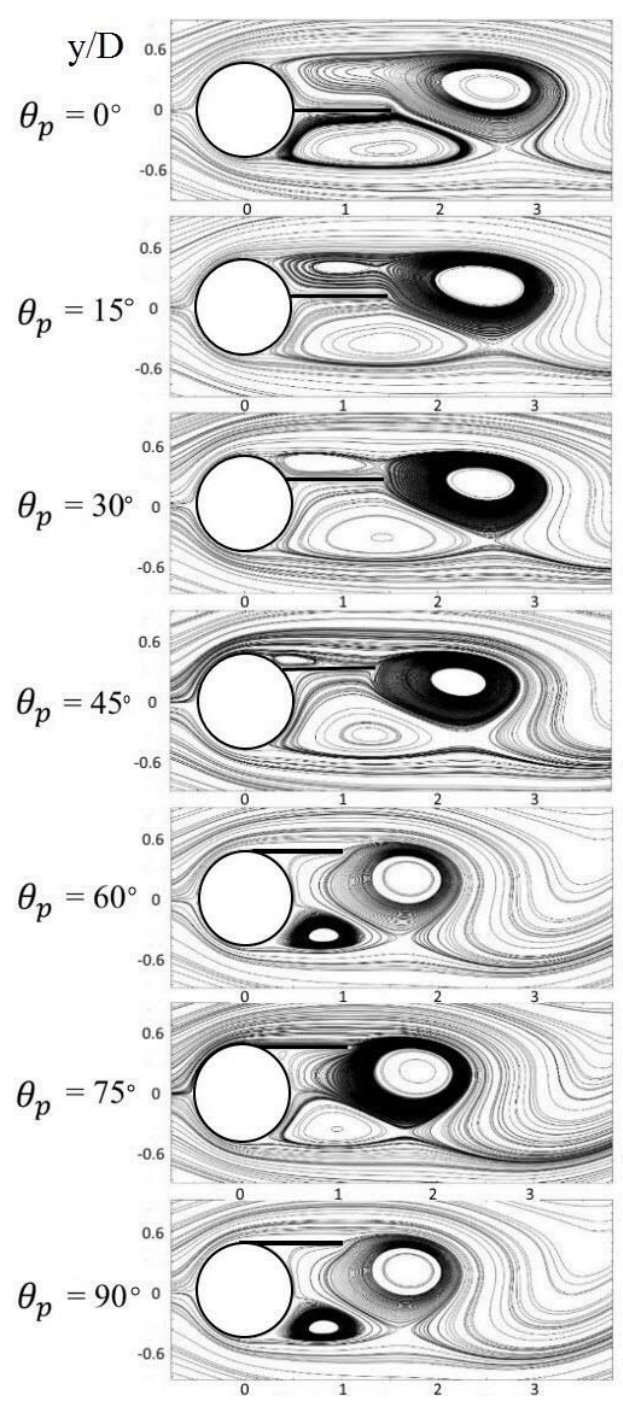

(a)
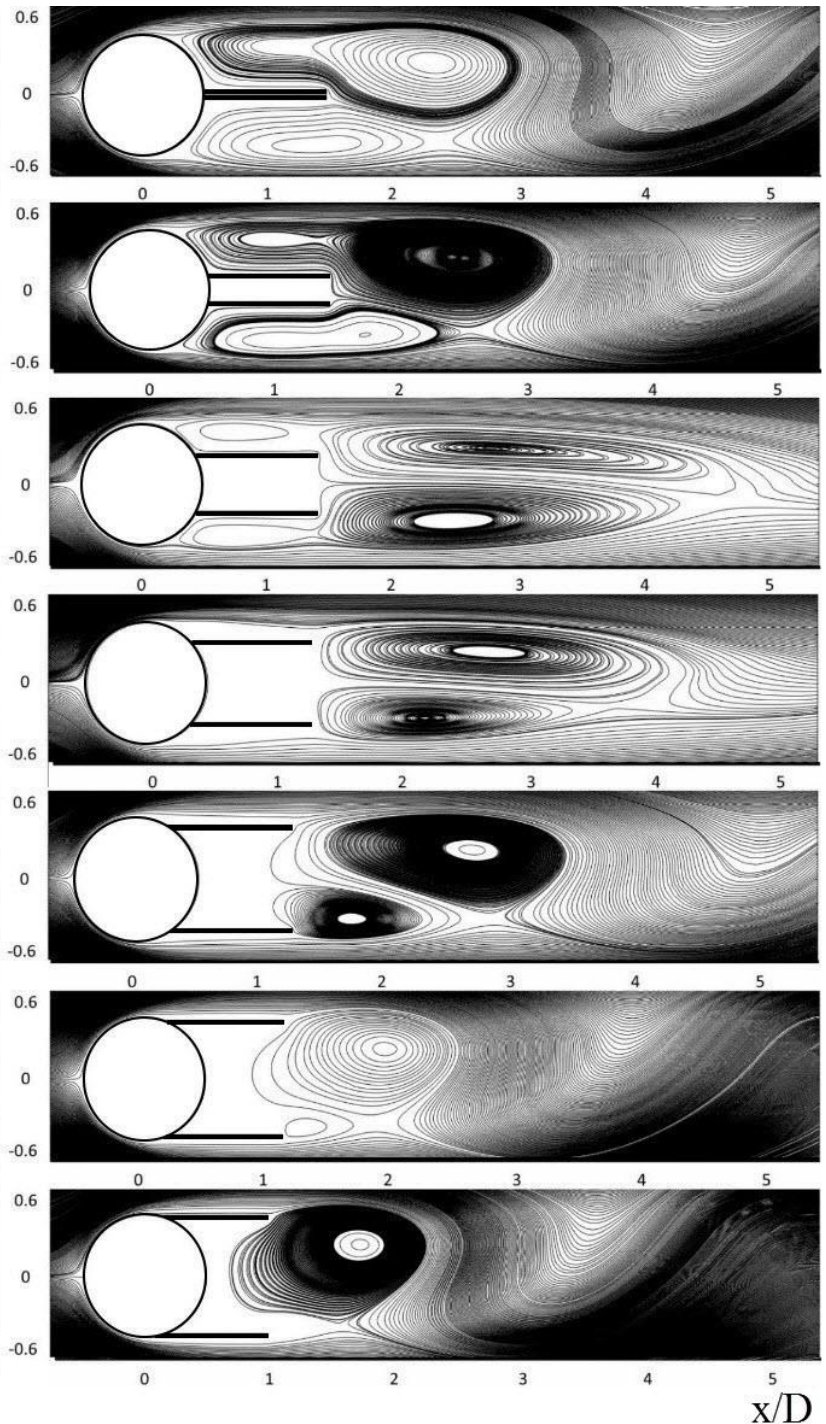

(b)

Figure 5: Instantaneous streamlines (a) cylinder with single-splitter (b) cylinder with dual-splitters.

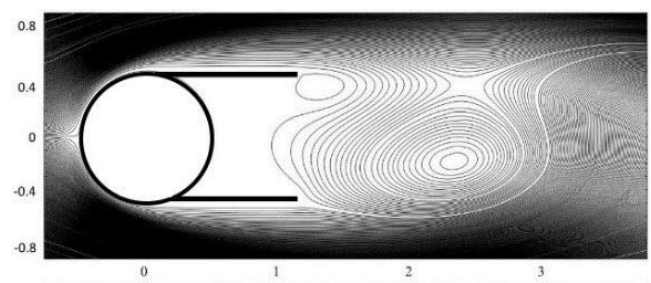

(a)

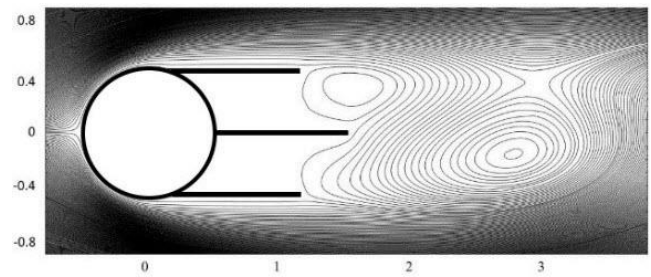

(b)

Figure 6: Instantaneous streamlines before lower vortex sheds at $\theta_{p}=75^{\circ}$ (a) cylinder with dualsplitters (b) cylinder with tri- splitters. 


\subsection{Mean drag force and fluctuating lift force}

Figure 7 shows the mean drag coefficient, $\overline{C_{D}}$, which was numerically calculated for the different considered cases. $\overline{C_{D}}$ in figure 7(a) was computed from integration of pressure and viscous stresses over the cylinder surface only, while $\overline{C_{D t}}$ in figure 7(b) was computed from integration over both the cylinder and plates walls. Moreover, the reference results of drag coefficient for the plain cylinder were incorporated into the figures for comparison. It can be seen from figures $7 \mathrm{a}$ and $7 \mathrm{~b}$ that a significant reduction in drag coefficient was achieved by the use of splitter plates in all the three cases compared to the plain cylinder. It can also be seen that dual-splitters and tri-splitters cases had lower values of drag coefficient than the single-splitter case.

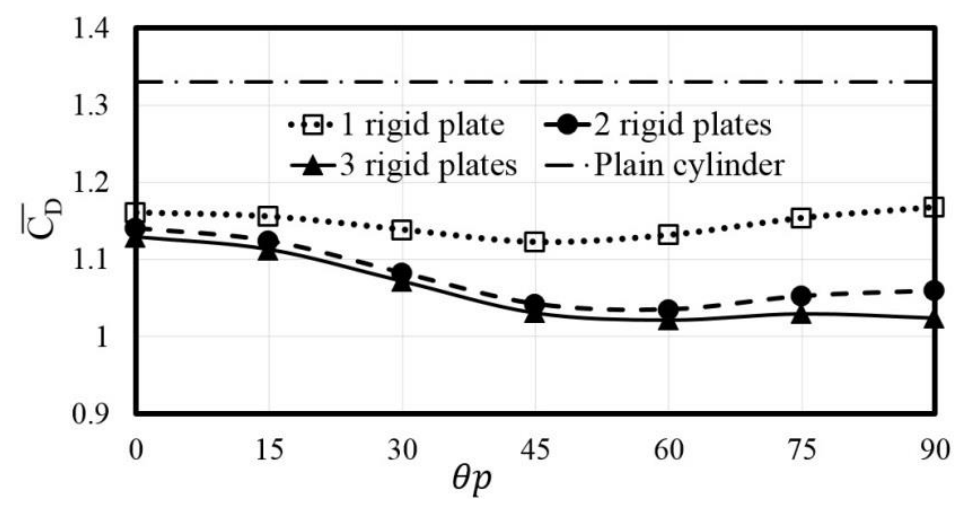

(a)

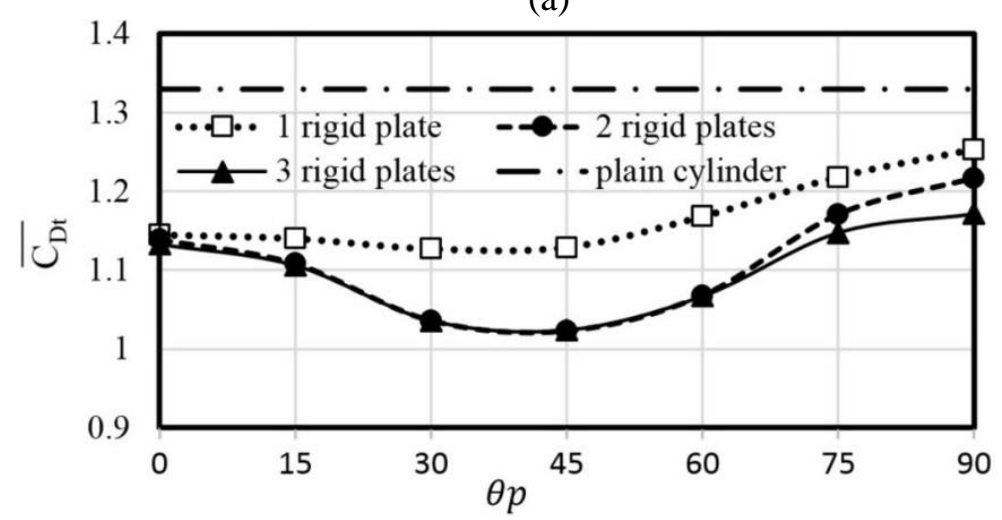

(b)

Figure 7: Mean drag coefficient, (a) $\overline{C_{D}}$ and (b) $\overline{C_{D t}}$, against the attachment angle of the splitter plates, $\theta_{p}$.

It can also be seen from figure 7(a) that in the case of the cylinder with one splitter plate, by increasing $\theta_{p}, \overline{C_{D}}$ remained almost constant within $0^{\circ} \leq \theta_{p} \leq 15^{\circ}$. Then, $\overline{C_{D}}$ decreased until it reached a minimum value at $\theta_{p}=45^{\circ}$. In this position the maximum drag reduction of $15 \%$ was attained compared to the plain cylinder. After that, by further increasing $\theta_{p}$ up to $90^{\circ}, \overline{C_{D}}$ increased up to almost its initial value at $0^{\circ}$. In the dual-splitters case, by increasing $\theta_{p}$ up to $45^{\circ}$, a similar trend as the single-splitter plate case can be observed between $\theta_{p}=0^{\circ}$ and $\theta_{p}=45^{\circ}$. However, the value of $\overline{C_{D}}$ of the cylinder with dual-splitters was considerably smaller than the one with a single-splitter plate, especially within $45^{\circ} \leq \theta_{p} \leq 90^{\circ}$. In this case, a maximum drag reduction of $23 \%$ compared to the plain cylinder case was observed at $\theta_{p}=45^{\circ}$. Our numerical result for drag coefficient at $\theta_{p}=40^{\circ}\left(\overline{C_{D}}=1.210\right)$ matches 
closely with that obtained by Bao and Tao (2013) $\left(\overline{C_{D}}=1.215\right)$ for the same dual-splitter configuration. In the case of cylinder with tri-splitters as can be seen in figure 7(a), the $\overline{C_{D}}$ was very close to that of the dual-splitters, showing that adding the third plate (middle plate in tri-splitter plate case) didn't have considerable effect on the drag coefficient.

It can be seen from figure $7(\mathrm{~b})$ that a similar trend as $\overline{C_{D}}$ was observed for $\overline{C_{D t}}$ in all three cases between $\theta_{p}=0^{\circ}$ and $\theta_{p}=45^{\circ}$. However, by further increasing $\theta_{p}, \overline{C_{D t}}$ had a higher rate of growth compared to $\overline{C_{D}}$ between $\theta_{p}=60^{\circ}$ and $\theta_{p}=90^{\circ}$ in all the simulated cases. This observation can be attributed to viscosity effect of the plates which has an increasing trend for $\theta_{p}>60^{\circ}$. As can be seen from figures 5(a) and 5(b), in low attachment angles $\left(0^{\circ} \leq \theta_{p} \leq 45^{\circ}\right)$, a vortex is formed between the plate and the main stream, reducing the effect of viscous drag due to the lack of contact between the surfaces of the plates and the main stream. In this position, the viscous component of the drag coefficient is expected to be significantly smaller than the pressure component. Hence, the plots of $\overline{C_{D}}$ and $\overline{C_{D t}}$ (figures $7 \mathrm{a}$ and $7 \mathrm{~b}$ ) share the same profile in this region. However, by increasing the attachment angle, especially when $\theta_{p} \geq 60^{\circ}$, the viscous component of the drag coefficient becomes more effective due to the contact between the plates surfaces and the main stream. Therefore, the plots of $\overline{C_{D}}$ and $\overline{C_{D t}}$ have different trends at $\theta_{p} \geq 60^{\circ}$. Consequently, dual-splitters are more influenced by this effect than a single-splitter. However, attaching third plate in the middle (tri-splitters case), doesn't cause significant change in viscous drag, due to the lack of effective contact between the surfaces of the middle plate and the main flow.

Figure 8 shows the root-mean-square (r.m.s.) value of the fluctuating lift coefficient $\left(C_{L}^{\prime}\right)$ which represents the influence of the splitter plates on the wake oscillation against attachment angle, $\theta_{p}$. The corresponding value of $C^{\prime}{ }_{L}$ for plain cylinder is incorporated to the figure for comparison. As can be seen from figure 8 , by changing the position of the plates, $C^{\prime}{ }_{L}$ has a similar trend as drag coefficient studied earlier in this section. Hence, the use of splitter plates attached to a circular cylinder can lead to reduction in wake oscillation. However, this reduction in dual-splitters and tri-splitters cases (which share similar profile) is more than the corresponding value of the cylinder with single-splitter. $C^{\prime}{ }_{L}$ had a decreasing trend by increasing the attachment angle within $0^{\circ} \leq \theta_{p} \leq 45^{\circ}$ and reached to its minimum at $\theta_{p}=45^{\circ}$ (in all three cases). At this position, a maximum reduction of $95 \%$ in $C_{L}^{\prime}$ was observed in dual-splitters and tri-splitters compared to $C^{\prime}{ }_{L}$ of the plain cylinder.

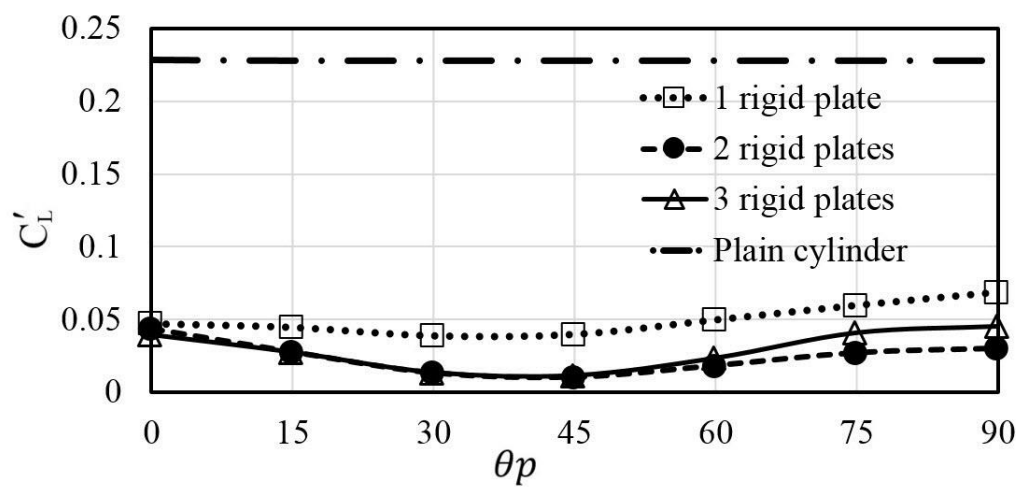

Figure 8: The r.m.s. value of the lift coefficient $\left(C^{\prime}{ }_{L}\right)$, against the attachment angle of the splitter plates, $\theta_{p}$. 
Also, it is interesting to see how much the average lift coefficient $\left(\overline{C_{L}}\right)$ is affected when using only one splitter at various positions. As can be seen from figure 9 , by increasing the attachment angle, $\overline{C_{L}}$ increased until it reached to its maximum value at $\theta_{p}=45^{\circ}$. By further increasing the attachment angle, $\overline{C_{L}}$ reduced and reached to zero at $\theta_{p} \approx 70^{\circ}$, and continued decreasing to negative values after that.

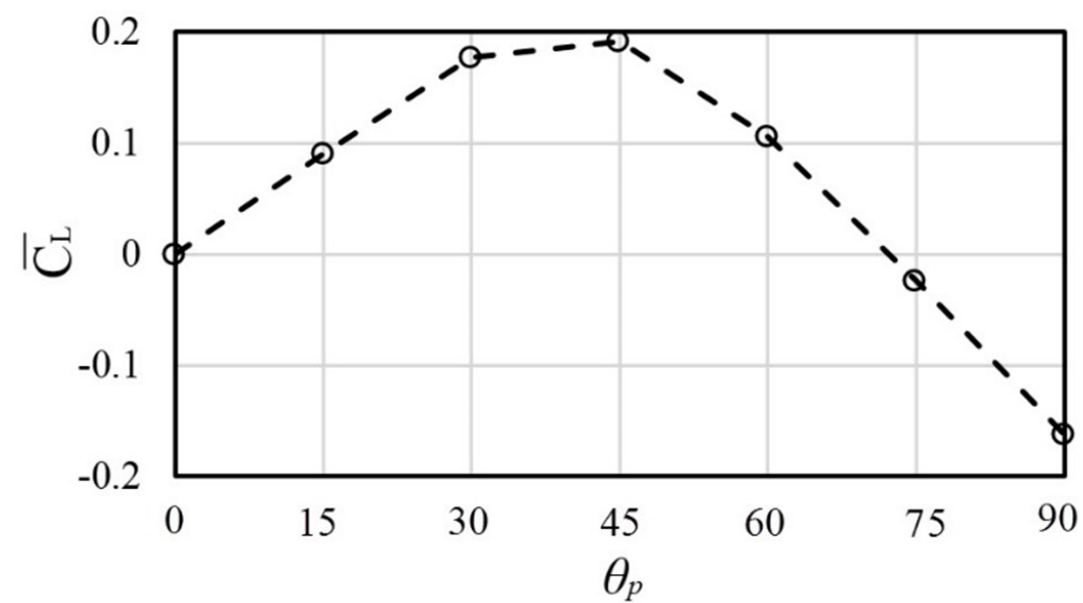

Figure 9: The average lift coefficient $\left(\overline{C_{L}}\right)$, against the attachment angle of the splitter plate, $\theta_{p}$, in cylinder with single-splitter.

\subsection{Pressure distribution on the cylinder surface}

The change in the drag coefficient and average lift coefficient investigated earlier in this paper are directly related to the pressure distribution. Figure 10 shows the mean pressure coefficient, $\overline{C p}$, against the angular position around the cylinder $(\theta)$ with one or two splitter plates attached at various positions $\left(\theta_{p}\right)$. For the single-splitter case because of the asymmetric configuration, the figure was plotted in the range of $-120^{\circ} \leq \theta \leq 120^{\circ}$ and for the dual splitters, due to the symmetric configuration, the figure was plotted in the range of $0^{\circ} \leq \theta \leq 120^{\circ}$. Outside $-120^{\circ} \leq \theta \leq 120^{\circ}$, curves shared the same profile; therefore, they are not plotted in the figures. It can be seen that in the single-splitter case (figure 10a) as well as the dual-splitters (figure 10b), the results are set into two regions of the curves. In the region between the wake centreline $(\theta=0)$ and the plate $\left(\theta=\theta_{p}\right)$, the pressure distribution was significantly influenced by the value of $\theta$, whereas in the other region $\left(\theta>\theta_{p}\right)$, the mean pressure coefficient had a similar trend against $\theta$. Therefore, we focused on the feature displayed within the region between the wake centreline and the plate $\left(0 \leq \theta \leq \theta_{p}\right)$. In this region, the values of $-\overline{C_{P}}$ for the cylinder with single-splitter (figure 10a) and cylinder with dual-splitters (figure 10b) for all plate positions (attachment angles) were smaller than the associated value of the plain cylinder. Because of that, the values of $\overline{C_{D}}$ in single-splitter and dual-splitters cases for all attachment angles were smaller that than $\overline{C_{D}}$ of the plain cylinder, as was seen in figure $7(a)$. 


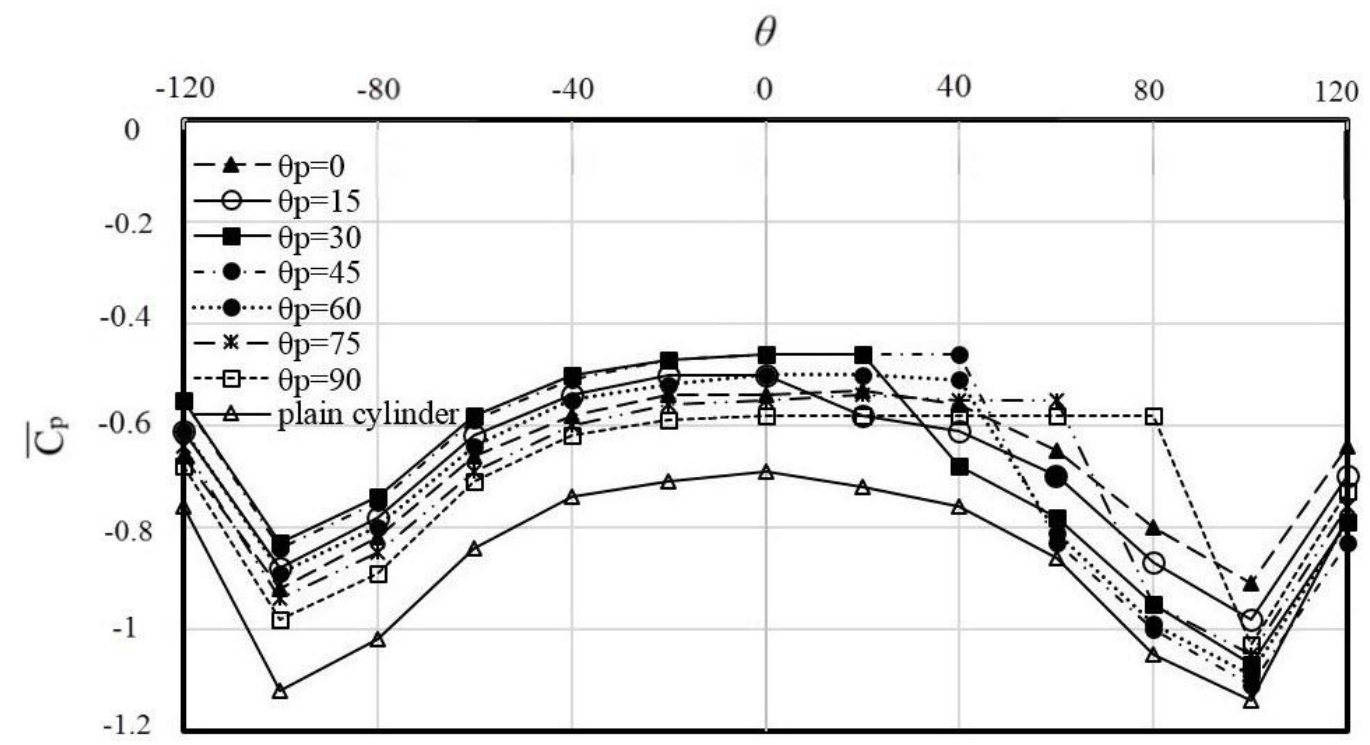

(a)

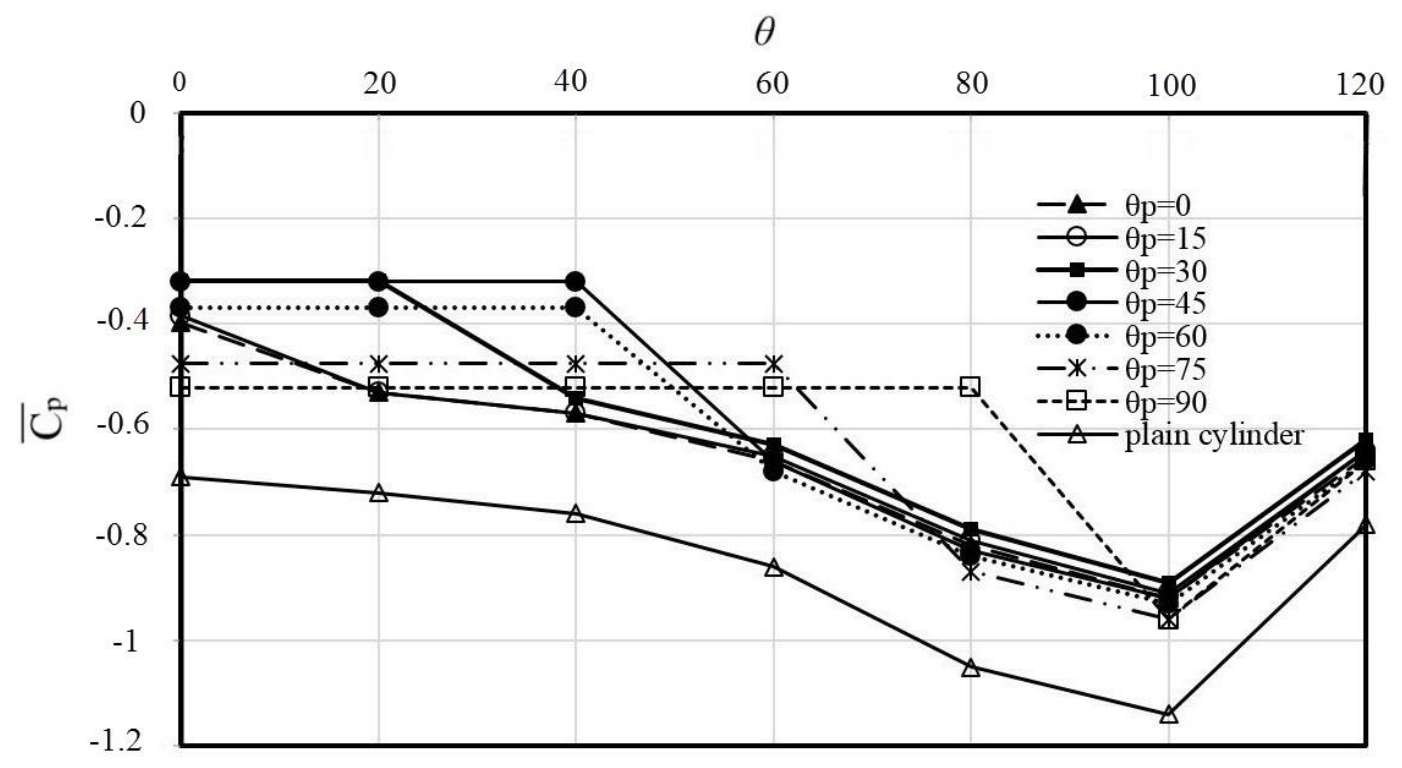

(b)

Figure 10: The mean pressure coefficient distribution, $\overline{C_{P}}$, against the angular position around the cylinder, $\theta$, in various attachment angle $\left(\theta_{p}\right)$ of splitter plates at downstream for (a) cylinder with single-splitter (b) cylinder with dual-splitters.

It can be seen in figure $10(\mathrm{~b})$ that in the case of cylinder with dual-splitters, $\overline{C_{P}}$ had a fixed value in the region between the wake centreline and the plate $\left(0<\theta<\theta_{p}\right)$. Similarly in trisplitters, $\overline{C_{P}}$ will have a fixed value in the region between the plates $\left(0<\theta<\theta_{p}\right)$. Therefore, the variation of $\overline{C_{D}}$ against attachment angle in the tri-splitters case can be justified by the behavior of the mean base pressure coefficient, $\overline{C_{P b}}\left(=\frac{P_{b}-P_{\infty}}{0.5 \rho U_{\infty}^{2}}\right)$ where $P_{b}$ is the static pressure on the cylinder surface at $\theta=0^{\circ}$. The variation of mean base pressure coefficient, $\overline{C_{P b}}$, against the attachment angle for the tri-splitters case is illustrated in figure 11. Moreover, the 
value of $\overline{C_{P b}}$ in the case of cylinder with dual-splitters, as well as the plain cylinder is incorporated into the figure for comparison. It can be seen that the plots of the dual-splitters and tri-splitters share almost the same profile within $0^{\circ} \leq \theta_{p} \leq 75^{\circ}$. Therefore, the value of $\overline{C_{D}}$ in tri-splitters is close to that in dual-splitters in this range (figure 7a). However, by further increasing $\theta_{p},-\overline{C_{P b}}$ in tri-splitters becomes smaller than that in dual-splitters; thus resulting in a lower value of $\overline{C_{D}}$ compared to that in dual-splitters.

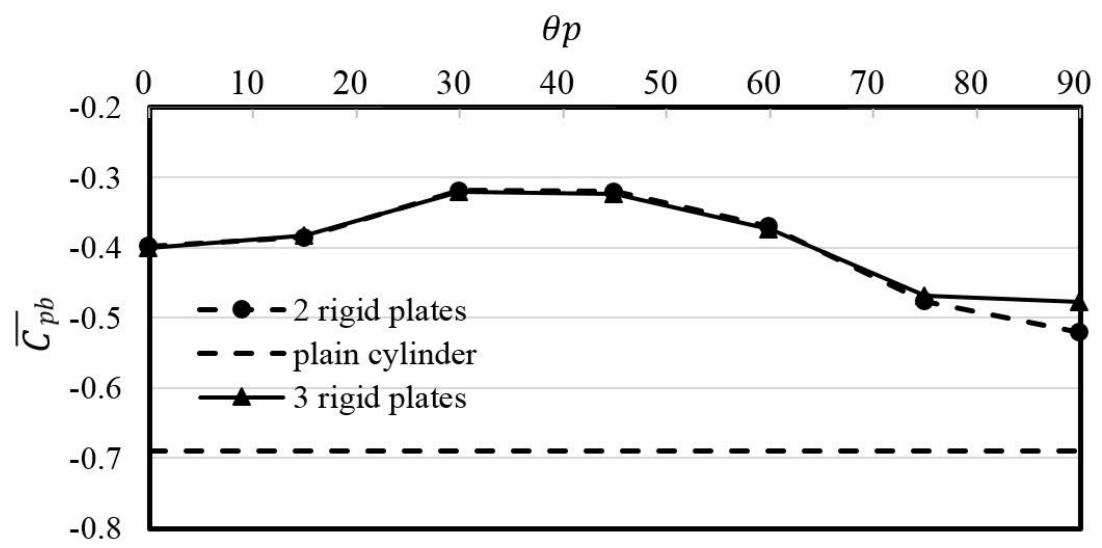

Figure 11: The mean base pressure coefficient, $\overline{C_{P b}}$, against attachment angle, $\theta_{p}$, in tri-splitters case compared with the plain cylinder and cylinder with two splitter plates.

\subsection{The effect of the length of middle plate in tri-splitters}

The effect of the plate length on flow quantities for single-splitter was investigated in a number of previous studies (Apelt and West, 1975; Apelt et al., 1973; Bearman, 1965; Kwon and Choi, 1996). Findings showed that the cylinder wake was highly influenced by the length of the splitter plate. In section 4.2 of the paper, it was observed that adding the third splitter plate of the same length, didn't have significant effect on the drag coefficient when compared with dual-splitters. In this section, in order to clarify the effect of adding the third plate on flow quantities, the length of the middle plate $\left(L_{m i d}\right)$ in tri-splitters was changed within $0 \leq L_{\text {mid }} / D \leq 4$ at a fixed attachment angle of $45^{\circ}$ which is associated with the position of two lateral splitters where the minimum value of drag was observed. Two different scenarios were considered based on the length of the lateral plates $\left(L_{\text {lat }}\right)$; tri-splitters with two long lateral plates of size $L_{l a} / D=1$, and tri-splitters with two short lateral plates of size $L_{l a t} / D=0.3$. Figure 12 shows the change in drag coefficient by varying the length of the middle plate $\left(L_{m i d}\right)$. It can be seen that with long lateral plates, the change in drag coefficient against the length of the middle plate was not noticeable. This is due to the fact that in this situation, the middle plate is located in a zone with no wake. However, when short lateral plates were utilized $\left(L_{\text {lat }} / D=0.3\right)$, it can be seen that increasing the length of the middle plate led to reduction of drag coefficient. In this case a maximum reduction of $7 \%$ was achieved when size of the middle plate was $L_{\text {mid }} / D=2$. However, further increase in the length of the middle didn't have noticeable effect on the value of drag coefficient. 


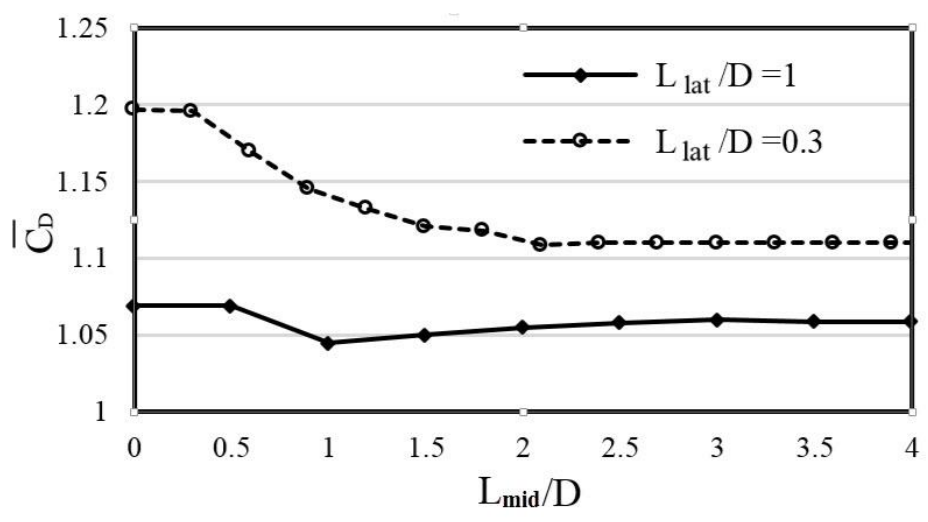

Figure 12: Mean drag coefficient, $\overline{C_{D}}$, against the dimensionless length of the middle plate $\left(L_{\text {mid }} / D\right)$ in two different cases: $L_{l a l} / D=0.3$ and $L_{l a l} / D=1$.

Figure 13 shows r.m.s. value of the lift coefficient $\left(C^{\prime}{ }_{L}\right)$ against the dimensionless length of the middle plate $\left(L_{\text {mid }} / D\right)$. It can be seen that when short lateral plates $\left(L_{l a l} / D=0.3\right)$ were used, by increasing the length of the middle plate up to $L_{\text {mid }} / D=2.1, C^{\prime}{ }_{L}$ has significantly decreased. In this case, increasing the length of the middle plate beyond $L_{\text {mid }} / D=2.1$ has resulted in complete prevention of the wake oscillations. However, $C^{\prime}{ }_{L}$ of tri-splitters with long lateral plates is considerably lower than the corresponding value of tri-splitters with short lateral plates. In this case, increasing the length of the middle plate beyond $L_{\text {mid }} / D=1.5$ has resulted in complete prevention of the wake oscillations.

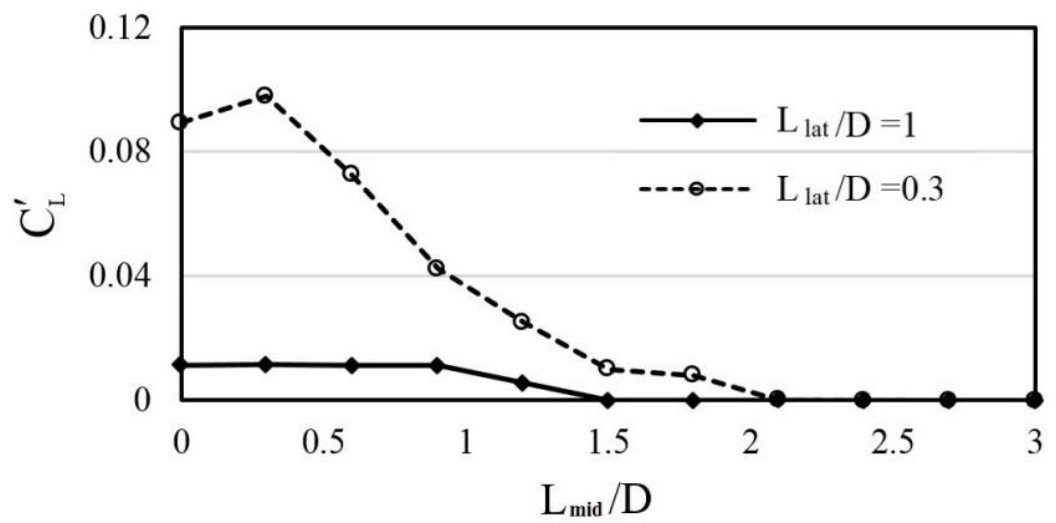

Figure 13: r.m.s. value of the lift coefficient $\left(C^{\prime}{ }_{L}\right)$, against the dimensionless length of the middle plate $\left(L_{\text {mid }} / D\right)$ in two different cases: $L_{l a} / D=0.3$ and $L_{l a} / D=1$.

The variation of mean base pressure coefficient, $\overline{C_{P b}}$, against the dimensionless length of the middle plate $\left(L_{\text {mid }} / D\right)$ in tri-splitters is illustrated in figure 14 . It can be seen that in the case of tri-splitters with short lateral plates $\left(L_{l a t} / D=0.3\right)$, by increasing the length of the middle plate, $-\overline{C_{P b}}$ has decreased and resulted in a decrease in $\overline{C_{D}}$ (as was seen in figure 12). However, in the case of tri-splitters with long lateral plates $\left(L_{l a t} / D=1\right)$, there was no considerable change in $-\overline{C_{P b}}$ by increasing $L_{\text {mid }}$, similar to what was observed for the corresponding $\overline{C_{D}}$ in figure 12 . 


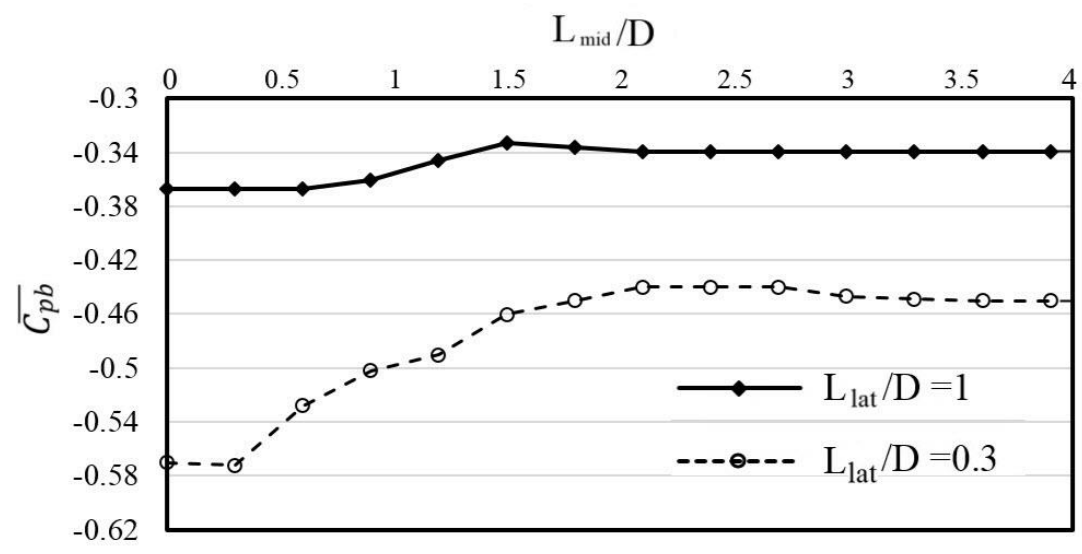

Figure 14: The mean base pressure coefficient, $\left(\overline{C_{P b}}\right)$, against the dimensionless length of the middle plate $\left(L_{\text {mid }} / D\right)$ in two different cases: $L_{l a l} / D=0.3$ and $L_{l a l} / D=1$.

\section{Summary and Conclusions}

A numerical investigation of the flow over a cylinder with up to three attached rigid splitter plates was performed in this work and the time-averaged characteristics were measured. The length of the plates was initially chosen to be equal to the cylinder diameter and the position of the plates was described by their attachment angle which was varied between $0^{\circ}$ and $90^{\circ}$. The main findings are as follows:

- Increasing the number of attached plates, from one to two, had a crucial effect on reduction of drag coefficient, Strouhal number and r.m.s. value of the lift coefficient. However, further increase to three plates, didn't have a significant effect on these quantities.

- The minimum drag coefficient was observed in the attachment angle of $45^{\circ}$. In this position, $15 \%$ reduction of drag coefficient was achieved for the cylinder with one attached splitter plate, and $23 \%$ reduction was achieved for the cylinders with two and three attached splitter plates, compared to the drag coefficient of a plain cylinder.

- In dual-splitters and tri-splitters cases, a maximum of $95 \%$ reduction in r.m.s. value of the lift coefficient compared to the corresponding value of the plain cylinder was achieved at $\theta_{p}=45^{\circ}$.

- In single-splitter plate case, the average value of lift coefficient was highly dependent on the position of the attached plate and the maximum value was observed at $\theta_{p} \approx$ $45^{\circ}$.

- In the single-splitter case, the minimum St was observed in the attachment angle of approximately $0^{\circ}$ which resulted in $17 \%$ reduction of $\mathrm{St}$ compared to the plain cylinder. In the dual-splitters and tri-splitters cases, the minimum St was observed in the attachment angle of $30^{\circ}$ in which $22 \%$ reduction of St was achieved compared to the plain cylinder.

- Although in tri-splitters case initially investigated $(L / D=1)$, the effect of the middle plate on flow quantities was not considerable, it was observed that when lateral plates are short, for instance $L_{l a t} / D=0.3$, the effect of the length of the middle plate on controlling the flow past the cylinder becomes noticeable. 
To sum up, the results of this study suggest that drag on cylinder, vortex shedding frequency and fluctuations of lift coefficient can be effectively reduced by attaching one or more splitter plates placed in an appropriate position. Assuming splitters are of the same length, the use of two parallel splitters symmetrically attached to the cylinder is recommended. The current investigation sheds light on the future developments of efficient drag controlling methods for a cylinder immersed in a free stream.

\section{References}

Akilli, H., Sahin, B., \& Tumen, N. F. (2005). Suppression of vortex shedding of circular cylinder in shallow water by a splitter plate. Flow Measurement and Instrumentation, 16(4), 211-219.

Apelt, C. J., West, G. S., \& Szewczyk, A. A. (1973). The effects of wake splitter plates on the flow past a circular cylinder in the range $104<\mathrm{R}<5 \times 10$ 4. Journal of Fluid Mechanics, 61(01), 187-198.

Apelt, C. J., \& West, G. S. (1975). The effects of wake splitter plates on bluff-body flow in the range $104<\mathrm{R}<5 \times 104$. Part 2. Journal of Fluid Mechanics, 71(01), 145-160.

Assi, G. R., Bearman, P. W., \& Kitney, N. (2009). Low drag solutions for suppressing vortex-induced vibration of circular cylinders. Journal of Fluids and Structures, 25(4), 666-675.

Bao, Y., \& Tao, J. (2013). The passive control of wake flow behind a circular cylinder by parallel dual plates. Journal of Fluids and Structures, 37, 201-219.

Barman, B. and Bhattacharyya, S., 2015. Control of vortex shedding and drag reduction through dual splitter plates attached to a square cylinder. Journal of Marine Science and Application, 14(2), pp.138-145.

Bearman, P. W. (1965). Investigation of the flow behind a two-dimensional model with a blunt trailing edge and fitted with splitter plates. Journal of Fluid Mechanics, 21(02), 241-255

Brown, P. N., Hindmarsh, A. C., \& Petzold, L. R. (1994). Using Krylov methods in the solution of large-scale differential-algebraic systems. SIAM Journal on Scientific Computing, 15(6), 1467-1488.

Comsol Multiphysics v. 4.3a, User's Manual, 2012.

Gerrard, J. H. (1966). The mechanics of the formation region of vortices behind bluff bodies. Journal of Fluid Mechanics, 25(02), 401-413.

Gu, F., Wang, J. S., Qiao, X. Q., \& Huang, Z. (2012). Pressure distribution, fluctuating forces and vortex shedding behavior of circular cylinder with rotatable splitter plates. Journal of fluids and structures, 28, 263-278.

Hindmarsh, A. C., Brown, P. N., Grant, K. E., Lee, S. L., Serban, R., Shumaker, D. E., \& Woodward, C. S. (2005). SUNDIALS: Suite of nonlinear and differential/algebraic equation solvers. ACM Transactions on Mathematical Software (TOMS), 31(3), 363-396.

Hwang, J. Y., Yang, K. S., \& Sun, S. H. (2003). Reduction of flow-induced forces on a circular cylinder using a detached splitter plate. Physics of Fluids (1994-present), 15(8), 2433-2436.

Kwon, K., \& Choi, H. (1996). Control of laminar vortex shedding behind a circular cylinder using splitter plates. Physics of Fluids (1994-present), 8(2), 479-486.

De Nayer, G., \& Breuer, M. (2014). Numerical FSI investigation based on LES: Flow past a cylinder with a flexible splitter plate involving large deformations (FSI-PfS-2a). International Journal of Heat and Fluid Flow, 50, 300-315.

Ozono, S. (2003). Vortex suppression of the cylinder wake by deflectors. Journal of wind engineering and industrial aerodynamics, 91(1), 91-99.

Park, J., Kwon, K., \& Choi, H. (1998). Numerical solutions of flow past a circular cylinder at Reynolds numbers up to 160. KSME international Journal, 12(6), 1200-1205. 
Posdziech, O., \& Grundmann, R. (2007). A systematic approach to the numerical calculation of fundamental quantities of the two-dimensional flow over a circular cylinder. Journal of Fluids and Structures, 23(3), 479-499.

Qiu, Y., Sun, Y., Wu, Y. and Tamura, Y., 2014. Effects of splitter plates and Reynolds number on the aerodynamic loads acting on a circular cylinder. Journal of Wind Engineering and Industrial Aerodynamics, 127, pp.40-50.

Roshko, A. (1954). On the drag and shedding frequency of two-dimensional bluff bodies (No. TN3169). Washington, DC: National Aeronautics and Space Administration.

Roshko, A. (1955). On the Wake and Drag of Bluff Bodies. Journal of the Aeronautical Sciences, Vol. 22, No. 2, 124-132.

Schenk, O., \& Gärtner, K. (2004). Solving unsymmetric sparse systems of linear equations with PARDISO. Future Generation Computer Systems, 20(3), 475-487.

Schenk, O., \& Gärtner, K. (2006). On fast factorization pivoting methods for sparse symmetric indefinite systems. Electronic Transactions on Numerical Analysis, 23(1), 158-179.

Serson, D., Meneghini, J. R., Carmo, B. S., Volpe, E. V., \& Assi, G. R. S. (2015). Numerical study of the flow around a circular cylinder with dual parallel splitter plates. In Instability and Control of Massively Separated Flows (pp. 169-174). Springer International Publishing.

Shukla, S., Govardhan, R. N., \& Arakeri, J. H. (2009). Flow over a cylinder with a hinged-splitter plate. Journal of Fluids and Structures, 25(4), 713-720.

Shukla, S., Govardhan, R. N., \& Arakeri, J. H. (2013). Dynamics of a flexible splitter plate in the wake of a circular cylinder. Journal of Fluids and Structures, 41, 127-134.

Sudhakar, Y., \& Vengadesan, S. (2012). Vortex shedding characteristics of a circular cylinder with an oscillating wake splitter plate. Computers \& Fluids, 53, 40-52.

Tian, F. B., Luo, H., Zhu, L., Liao, J. C., \& Lu, X. Y. (2011). An efficient immersed boundary-lattice Boltzmann method for the hydrodynamic interaction of elastic filaments. Journal of computational physics, 230(19), 7266-7283.

Unal, M. F., \& Rockwell, D. (1988). On vortex formation from a cylinder. Part 1. The initial instability. Journal of Fluid Mechanics, 190, 491-512.

Williamson, C. H. K. (1989). Oblique and parallel modes of vortex shedding in the wake of a circular cylinder at low Reynolds numbers. Journal of Fluid Mechanics, 206, 579-627.

Yucel, S. B., Cetiner, O., \& Unal, M. F. (2010). Interaction of circular cylinder wake with a short asymmetrically located downstream plate. Experiments in Fluids, 49(1), 241-255. 